\title{
A reconsideration of Jensen's inequality and its applications
}

\section{Yasuo Nakasuji ${ }^{1}$ and Sin-Ei Takahasi ${ }^{*}$}

\section{${ }^{*}$ Correspondence:}

sin_ei1@yahoo.co.jp

2Toho University, Funabashi

274-8510, Japan

Full list of author information is

available at the end of the article

\begin{abstract}
A finite form of Jensen's inequality for a continuous convex function from a topological abelian semigroup to another topological ordered abelian semigroup is obtained under some assumption. As an application, a refinement of a mean inequality is also obtained.
\end{abstract}

MSC: Primary 39B62; secondary 26B25; 26A51

Keywords: Jensen's inequality; mean; refinement; continuous associative semigroup operation

\section{Introduction}

This is the inheritance of the idea of [1, Theorem 1] which gives a new interpretation of Jensen's inequality by $\varphi$-mean. The finite form of Jensen's inequality proved by Jensen in 1906 asserts that if $t_{1}, \ldots, t_{n}$ are positive numbers with $\sum_{i=1}^{n} t_{i}=1$ and $f$ is a continuous convex (resp. concave) function on a real interval $I$, then

$$
f\left(\sum_{i=1}^{n} t_{i} x_{i}\right) \leq \sum_{i=1}^{n} t_{i} f\left(x_{i}\right) \quad\left(\text { resp. } f\left(\sum_{i=1}^{n} t_{i} x_{i}\right) \geq \sum_{i=1}^{n} t_{i} f\left(x_{i}\right)\right)
$$

holds for all $x_{1}, \ldots, x_{n} \in I$.

We first introduce a concept called ' $(*, \circ)$-convex (or concave)' for a continuous function from a topological abelian semigroup $(I, *)$ to another topological ordered abelian semigroup $(J, \circ)$ and give an interesting example of such a function (see Remark 1 ). Our purpose of this paper is to give a finite form of Jensen's inequality for such a function under some assumption (see Theorem 1). Also, as an application, we give a refinement of a mean inequality (see Theorem 2).

\section{Terminology and main theorem}

Let $I$ be a topological space, and let $*$ be a topological abelian semigroup operation on $I$. For any $x \in I$ and $n \in \mathbf{N}$ with $n \geq 1$, define the $n$th power $x^{(n)}$ of $x$ recursively by $x^{(1)_{*}}=x$ and $x^{(n+1)_{*}}=x^{(n)} * * x$ for $n \geq 1$. We assume that

$\left(\sharp_{1}\right)$ any $n$ th-power function $x \mapsto x^{(n)} *$ is a bijection of $I$ onto itself.

\section{照 Springer}

(c) 2013 Nakasuji and Takahasi; licensee Springer. This is an Open Access article distributed under the terms of the Creative Commons Attribution License (http://creativecommons.org/licenses/by/2.0), which permits unrestricted use, distribution, and reproduction in any medium, provided the original work is properly cited. 
By the assumption $\left(\sharp_{1}\right)$, for each $x \in I$ and $n \in \mathbf{N}$, there exists a unique element $a$ of $I$ such that $a^{(n) *}=x$. Denote by $x^{(1 / n)_{*}}$ such an element $a$. Moreover, we define

$$
x^{(m / n) *}=\left(x^{(1 / n) *}\right)^{(m) *}
$$

for each $m, n \in \mathbf{N}$. Then we can easily see that this definition is well defined, that is,

$$
\frac{m}{n}=\frac{m^{\prime}}{n^{\prime}} \Rightarrow\left(x^{(1 / n)_{*}}\right)^{(m)_{*}}=\left(x^{\left(1 / n^{\prime}\right)_{*}}\right)^{\left(m^{\prime}\right)_{*}} \quad(\forall x \in I) .
$$

In this case, we can easily show that the following power laws:

$$
x^{(p+q)_{*}}=x^{(p)_{*}} * x^{(q)_{*}}, \quad x^{(p q)_{*}}=\left(x^{(p)_{*}}\right)^{(q)_{*}} \quad \text { and } \quad(x * y)^{(p)_{*}}=x^{(p)_{*} * y^{(p)_{*}}}
$$

for all $p, q \in \mathbf{Q}_{+}$and $x, y \in I$. Here $\mathbf{Q}_{+}$denotes the set of all positive rational numbers. Moreover, we assume that

$\left(\sharp_{2}\right)$ for each $x \in I$, the function $p \mapsto x^{(p) *}$ is continuous on $\mathbf{Q}_{+}$and it has a continuous extension to $\mathbf{R}_{+}$, say $t \mapsto x^{(t)_{*}}$.

Here $\mathbf{R}_{+}$denotes the set of all positive real numbers. Therefore power laws (1) hold for all $p, q \in \mathbf{R}_{+}$. Denote by $\mathcal{A}_{+}(I)$ the set of all topological abelian semigroup operations on $I$ satisfying the assumptions $\left(\sharp_{1}\right)$ and $\left(\sharp_{2}\right)$. Our assumption $\left(\sharp_{1}\right)$ leads to the following important concept called 'mean'. For each $x, y \in I$, put

$$
M_{*}(x, y)=(x * y)^{(1 / 2)_{*}} .
$$

We call $M_{*}(x, y)$ the mean of $x$ and $y$ with respect to the operation $*$.

Moreover, let $J$ be a topological ordered space with relation $\leq$, and denote by $\mathcal{A}_{+}^{0}(J, \leq)$ the set of all operations $\circ \in \mathcal{A}_{+}(J)$ such that

$\left(b_{1}\right) a \leq b \Leftrightarrow a \circ c \leq b \circ c(a, b, c \in J)$

and

$\left(b_{2}\right) a \leq b \Rightarrow a^{(t)_{\circ}} \leq b^{(t)_{\circ}}\left(a, b \in J, t \in \mathbf{R}_{+}\right)$.

Let $C(I, J)$ be the set of all continuous functions from $I$ to $J$. Take $* \in \mathcal{A}_{+}(I)$, $\circ \in \mathcal{A}_{+}^{0}(J, \leq)$ and $f \in C(I, J)$ arbitrarily. If $f$ satisfies

$$
f\left(M_{*}(x, y)\right) \leq M_{\circ}(f(x), f(y)) \quad\left(\text { resp. } f\left(M_{*}(x, y)\right) \geq M_{\circ}(f(x), f(y))\right)
$$

for all $x, y \in I$, then we say that $f$ is said to be $(*, \circ)$-convex (resp. concave).

The following theorem states a finite form of Jensen's inequality for a $(*, 0)$-convex (or concave) function.

Theorem 1 Let $* \in \mathcal{A}_{+}(I)$ and $\circ \in \mathcal{A}_{+}^{0}(J, \leq)$. If $\in C(I, J)$ is $(*, \circ)$-convex, then

$$
f\left(x_{1}^{\left(t_{1}\right) *} * \cdots * x_{n}^{\left(t_{n}\right) *}\right) \leq f\left(x_{1}\right)^{\left(t_{1}\right)} \circ \cdots \circ f\left(x_{n}\right)^{\left(t_{n}\right) \circ}
$$

holds for all $n \in \mathbf{N}, x_{1}, \ldots, x_{n} \in I$ and $t_{1}, \ldots, t_{n} \in \mathbf{R}_{+}$with $t_{1}+\cdots+t_{n}=1$.

Iff is $(*, \circ)$-concave, then the inequality above is reversed. 
Remark 1 Let $\mathbf{R}_{+}^{2}$ be the product space of $\mathbf{R}_{+}$with ordinary topology. Let $*$ be the operation on $\mathbf{R}_{+}^{2}$ defined by

$$
(a, b) *(c, d)=(a c, a d+b c)
$$

for each $(a, b),(c, d) \in \mathbf{R}_{+}^{2}$. Then $*$ is a topological abelian semigroup operation on $\mathbf{R}_{+}^{2}(c f$. [2, p.157-160]). In fact, $\left(\mathbf{R}_{+}^{2}, *\right)$ is topologically isomorphic to an abelian subsemigroup of the semigroup of all $2 \times 2$ matrices with usual product under the following mapping:

$$
(a, b) \mapsto\left(\begin{array}{cc}
a & b \\
0 & a
\end{array}\right)
$$

Note that

$$
(a, b)^{(n)_{*}}=\left(a^{n}, n a^{n-1} b\right) \quad \text { and } \quad(a, b)^{(1 / n)_{*}}=\left(a^{1 / n}, \frac{b}{n a^{1-1 / n}}\right)
$$

for all $n \in \mathbf{N}$. Then a simple calculation implies that

$$
(a, b)^{(m / n)_{*}}=\left(a^{m / n}, \frac{m}{n} a^{\frac{m}{n}-1} b\right)
$$

for each $m, n \in \mathbf{N}$. Therefore we see that $* \in \mathcal{A}_{+}\left(\mathbf{R}_{+}^{2}\right)$. In this case, we obtain from a simple calculation that

$$
M_{*}((a, b),(c, d))=\left(\sqrt{a c}, \frac{a d+b c}{2 \sqrt{a c}}\right)
$$

for each $(a, b),(c, d) \in \mathbf{R}_{+}^{2}$.

Now let be the ordinary multiplication on $\mathbf{R}_{+}$. Since $\mathbf{R}_{+}$becomes a topological ordered space with the ordinary topology and the ordinary order $\leq$, we have that $\cdot \in \mathcal{A}_{+}^{0}\left(\mathbf{R}_{+}, \leq\right)$ and $M .(x, y)=\sqrt{x y}$ for each $x, y \in \mathbf{R}_{+}$. Let $\alpha$ and $\beta$ be real numbers and put

$$
f_{\alpha, \beta}(a, b)=a^{\alpha} b^{\beta}
$$

for each $(a, b) \in \mathbf{R}_{+}^{2}$. Then $f_{\alpha, \beta}$ is a continuous function from $\mathbf{R}_{+}^{2}$ to $\mathbf{R}_{+}$such that

$$
f_{\alpha, \beta}\left(M_{*}((a, b),(c, d))\right)=(\sqrt{a c})^{\alpha-\beta}\left(\frac{a d+b c}{2}\right)^{\beta}
$$

and

$$
M .\left(f_{\alpha, \beta}(a, b), f_{\alpha, \beta}(c, d)\right)=(\sqrt{a c})^{\alpha}(\sqrt{b d})^{\beta}
$$

for each $(a, b),(c, d) \in \mathbf{R}_{+}^{2}$. Therefore we can easily see that if $\beta \leq 0$ (resp. $\left.\beta \geq 0\right)$, then $f_{\alpha, \beta}$ is $(*, \cdot)$-convex (resp. $(*, \cdot)$-concave).

Remark 2 Let $E$ be a nontrivial real interval with the ordinary topology and the ordinary order $\leq$. In this case, Craigen and Pales [3] showed that if $\circ$ is a continuous cancellative 
semigroup operation on $E$, then there exists a continuous order-preserving bijection $\varphi$ of $E$ onto another (necessarily unbounded) real interval such that

$$
a \circ b=\varphi^{-1}(\varphi(a)+\varphi(b))
$$

for all $a, b \in E$ (cf. Aczel $[4,5])$. Therefore we can easily see that if $\varphi(E)=\mathbf{R}_{+}$, then all continuous cancellative semigroup operations on $E$ are in $\mathcal{A}_{+}^{0}(E, \leq)$.

Remark 3 It is clear that a direct product of $\mathbf{R}_{+}$admits a semigroup operation in $\mathcal{A}_{+}^{0}$ that is given as the product of semigroup operations on each $\mathbf{R}_{+}$. However, the semigroup operation on $\mathbf{R}_{+}^{2}$ described in Remark 1 does not satisfy properties $\left(b_{1}\right)$ and $\left(b_{2}\right)$. So, it would be of interest to give an example of an ordered abelian semigroup with a semigroup operation in $\mathcal{A}_{+}^{0}$, which is not isomorphic, as a topological semigroup, to the direct product of the topological semigroup $\mathbf{R}_{+}$.

\section{Lemmas and proof of Theorem 1}

Throughout this section, let $I$ and $J$ be as in Section 2 and suppose that $* \in \mathcal{A}_{+}(I)$, ० $\in$ $\mathcal{A}_{+}^{0}(J, \leq)$ and that $f \in C(I, J)$ is $(*, 0)$-convex.

Remark 4 If $f \in C(I, J)$ is $(*, \circ)$-concave, then all inequalities in this section are reversed.

Lemma 1 The inequality

$$
f\left(x_{1}^{(1 / 2)_{*}} * \cdots * x_{n}^{\left(1 / 2^{n}\right) *}\right) \leq f\left(x_{1}\right)^{(1 / 2) \circ} \circ \cdots \circ f\left(x_{n-1}\right)^{\left(1 / 2^{n-1}\right) \circ} \circ\left[f\left(x_{n}^{(1 / 2) *}\right)\right]^{\left(1 / 2^{n-1}\right) \circ}
$$

holds for all $n \in \mathbf{N}$ and $x_{1}, \ldots, x_{n} \in I$.

Proof Let $n \in \mathbf{N}$ and $x_{1}, \ldots, x_{n} \in I$. Since $f$ is $(*, \circ)$-convex, it follows that

$$
\begin{aligned}
& f\left(x_{1}^{(1 / 2) *} * x_{2}^{\left(1 / 2^{2}\right) *} * \cdots * x_{n}^{\left(1 / 2^{n}\right) *}\right) \\
& =f\left(\left(x_{1} * x_{2}^{(1 / 2) *} * \cdots * x_{n}^{\left(1 / 2^{n-1}\right) *}\right)^{(1 / 2) *}\right) \\
& \leq\left(f\left(x_{1}\right) \circ f\left(x_{2}^{(1 / 2)_{*}} * \cdots * x_{n}^{\left(1 / 2^{n-1}\right) *}\right)\right)^{(1 / 2) \text { 。 }} \\
& =f\left(x_{1}\right)^{(1 / 2)} \circ\left[f\left(x_{2}^{(1 / 2) *} * \cdots * x_{n}^{\left(1 / 2^{n-1}\right) *}\right)\right]^{(1 / 2) 。} \\
& =f\left(x_{1}\right)^{(1 / 2) \circ} \circ\left[f\left(\left(x_{2} * x_{3}^{(1 / 2) *} * \cdots * x_{n}^{\left(1 / 2^{n-2}\right) *}\right)^{(1 / 2) *}\right)\right]^{(1 / 2) 。} \\
& \leq f\left(x_{1}\right)^{(1 / 2) \circ} \circ\left[\left(f\left(x_{2}\right) \circ f\left(x_{3}^{(1 / 2) *} * \cdots * x_{n}^{\left(1 / 22^{n-2}\right) *}\right)\right)^{(1 / 2) \circ}\right]^{(1 / 2) 。} \\
& =f\left(x_{1}\right)^{(1 / 2) \circ} \circ f\left(x_{2}\right)^{\left(1 / 2^{2}\right) \circ} \circ\left[f\left(x_{3}^{(1 / 2) *} * \cdots * x_{n}^{\left(1 / 2^{n-2}\right) *}\right)\right]^{\left(1 / 2^{2}\right) \circ} \\
& \text {... } \\
& \leq f\left(x_{1}\right)^{(1 / 2) \circ} \circ f\left(x_{2}\right)^{\left(1 / 2^{2}\right) \circ} \circ \cdots \circ f\left(x_{n-1}\right)^{\left(1 / 2^{n-1}\right) \circ} \circ\left[f\left(x_{n}^{(1 / 2) *}\right)\right]^{\left(1 / 2^{n-1}\right) \circ} \text {. }
\end{aligned}
$$

Therefore we obtain the desired inequality.

Let $p$ and $q$ be two nonnegative rational numbers. We define $x^{(p)_{*}} * y^{(q) *}=y^{(q)_{*}}$ if $p=0$,

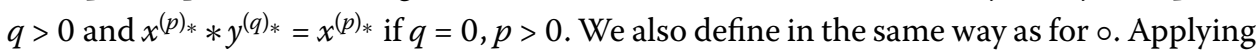
these notations, we show the following lemma. 
Lemma 2 The inequality $f\left(x^{(p)_{*}} * y^{(1-p)_{*}}\right) \leq f(x)^{(p)_{\circ}} \circ f(y)^{(1-p)_{\circ}}$ holds for all $x, y \in I$ and $p \in \mathbf{R}_{+}$with $0<p<1$.

Proof Let $x, y \in I$ and $p \in \mathbf{R}_{+}$with $0<p<1$. By the binary system, we have the expansion $p=\sum_{i=1}^{\infty} p_{i} / 2^{i}$, where $p_{i} \in\{0,1\}(i=1,2, \ldots)$. Since $p_{i} \in\{0,1\}(i=1,2, \ldots)$, it follows that

$$
\begin{aligned}
& \lim _{n \rightarrow \infty}\left[f\left(x^{\left(p_{1}\right) *} * y^{\left(1-p_{1}\right) *}\right)\right]^{(1 / 2) \circ} \circ \cdots \circ\left[f\left(x^{\left(p_{n-1}\right) *} * y^{\left(1-p_{n-1}\right) *}\right)\right]^{\left(1 / 2^{n-1}\right) 。} \\
& =\lim _{n \rightarrow \infty}\left[f(x)^{\left(p_{1} / 2\right) \circ} \circ f(y)^{\left(\left(1-p_{1}\right) / 2\right)_{\circ}}\right] \circ \cdots \\
& \circ\left[f(x)^{\left(p_{n} / 2^{n-1}\right) \circ} \circ f(y)^{\left(\left(1-p_{n-1}\right) / 2^{n-1}\right)}\right] \\
& =\lim _{n \rightarrow \infty} f(x)^{\left(\sum_{i=1}^{n-1} p_{i} / 2^{i}\right)} \circ f(y)^{\left(\sum_{i=1}^{n-1}\left(1-p_{i}\right) / 2^{i}\right)} \\
& =f(x)^{(p)} \circ f(y)^{(1-p)_{\circ}} \text {. }
\end{aligned}
$$

So, putting

$$
a_{n}=\left[f\left(x^{\left(p_{1}\right) *} * y^{\left(1-p_{1}\right) *}\right)\right]^{(1 / 2) \circ} \circ \cdots \circ\left[f\left(x^{\left(p_{n-1}\right) *} * y^{\left(1-p_{n-1}\right) *}\right)\right]^{\left(1 / 2^{n-1}\right) \circ}
$$

for each $n \in \mathbf{N}$, we have

$$
\lim _{n \rightarrow \infty} a_{n}=f(x)^{(p)_{\circ}} \circ f(y)^{(1-p)_{\circ}} .
$$

Put

$$
\mathbf{N}_{1}=\left\{n \in \mathbf{N}: f\left(\left(x^{\left(p_{n}\right)_{*}} * y^{\left(1-p_{n}\right)_{*}}\right)^{(1 / 2) *}\right)=f\left(x^{(1 / 2)_{*}}\right)\right\}
$$

and

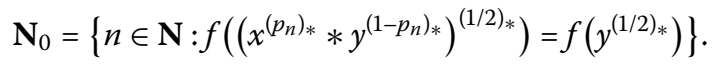

Since

$$
f\left(\left(x^{\left(p_{n}\right) *} * y^{\left(1-p_{n}\right) *}\right)^{(1 / 2) *}\right)= \begin{cases}f\left(x^{(1 / 2) *}\right) & \left(p_{n}=1\right), \\ f\left(y^{(1 / 2) *}\right) & \left(p_{n}=0\right)\end{cases}
$$

for each $n \in \mathbf{N}$, it follows that $\mathbf{N}=\mathbf{N}_{1} \cup \mathbf{N}_{0}$. Then either $\mathbf{N}_{1}$ or $\mathbf{N}_{0}$ are infinite. Put

$$
e_{n}=\left[f\left(\left(x^{\left(p_{n}\right) *} * y^{\left(1-p_{n}\right) *}\right)^{(1 / 2) *}\right)\right]^{\left(1 / 2^{n-1}\right)}
$$

for each $n \in \mathbf{N}$. If $\mathbf{N}_{1}$ is infinite, then we have

$$
\lim _{n \in \mathbf{N}_{1}} e_{n} \circ f\left(x^{(1 / 2)_{*}}\right)=\lim _{n \in \mathbf{N}_{1}}\left(f\left(x^{(1 / 2) *}\right)\right)^{\left(1+1 / 2^{n-1}\right) \circ}=f\left(x^{(1 / 2)_{*}}\right) .
$$

Therefore it follows from (2) and (3) that

$$
f(x)^{(p)_{\circ}} \circ f(y)^{(1-p)_{\circ}} \circ f\left(x^{(1 / 2)_{*}}\right)=\lim _{n \in \mathbf{N}_{1}} a_{n} \circ e_{n} \circ f\left(x^{(1 / 2)_{*}}\right) .
$$


Also we have from Lemma 1 that

$$
\begin{aligned}
& f\left(x^{\left(\sum_{i=1}^{n} p_{i} / 2^{i}\right) *} * y^{\left(\sum_{i=1}^{n}\left(1-p_{i}\right) / 2^{i}\right) *}\right) \\
&= f\left(\left(x^{\left(p_{1}\right) *} * y^{\left(1-p_{1}\right) *}\right)^{(1 / 2) *} * \cdots *\left(x^{\left(p_{n}\right) *} * y^{\left(1-p_{n}\right) *}\right)^{\left(1 / 2^{n}\right) *}\right) \\
& \leq {\left[f\left(x^{\left(p_{1}\right) *} * y^{\left(1-p_{1}\right) *}\right)\right]^{(1 / 2) \circ} \circ \cdots \circ\left[f\left(x^{\left(p_{n-1}\right) *} * y^{\left(1-p_{n-1}\right) *}\right)\right]^{\left(1 / 2^{n-1}\right) \circ} } \\
&\left.\circ\left[f\left(\left(x^{\left(p_{n}\right) *} * y^{\left(1-p_{n}\right) *}\right)\right)^{(1 / 2) *}\right)\right]^{\left(1 / 2^{n-1}\right) \circ} \\
&= a_{n} \circ e_{n}
\end{aligned}
$$

for all $n \in \mathbf{N}_{1}$. Then it follows from $\left(b_{1}\right)$ that

$$
f\left(x^{\left(\sum_{i=1}^{n} p_{i} / 2^{i}\right) *} * y^{\left(\sum_{i=1}^{n}\left(1-p_{i}\right) / 2^{i}\right) *}\right) \circ f\left(x^{(1 / 2)_{*}}\right) \leq a_{n} \circ e_{n} \circ f\left(x^{(1 / 2)_{*}}\right)
$$

for all $n \in \mathbf{N}_{1}$. Letting $n \in \mathbf{N}_{1} \rightarrow \infty$ in (5), we obtain from (4) that

$$
f\left(x^{(p)_{*}} * y^{(1-p)_{*}}\right) \circ f\left(x^{(1 / 2) *}\right) \leq f(x)^{(p) \circ} \circ f(y)^{(1-p) \circ} \circ f\left(x^{(1 / 2)_{*}}\right) .
$$

Canceling $f\left(x^{(1 / 2)_{*}}\right)$ in $(6)$ by $\left(b_{1}\right)$, we obtain the desired inequality. Similarly, the desired inequality is obtained in case that $\mathbf{N}_{0}$ is infinite.

Lemma 3 The inequality

$$
f\left(\left(x_{1} * \cdots * x_{n}\right)^{(1 / n) *}\right) \leq\left(f\left(x_{1}\right) \circ \cdots \circ f\left(x_{n}\right)\right)^{(1 / n) 。}
$$

holds for all $n \in \mathbf{N}$ and $x_{1}, \ldots, x_{n} \in I$.

Proof It is clear that the lemma holds for $n=1$. Suppose the lemma holds for $n=k$. Let $x_{1}, \ldots, x_{k}, x_{k+1} \in I$ and put

$$
a=\left(f\left(x_{1}\right) \circ \cdots \circ f\left(x_{k}\right)\right)^{(1 / k) \circ} \quad \text { and } \quad x=\left(x_{1} * \cdots * x_{k}\right)^{(1 / k)_{*}} .
$$

Then $a \geq f(x)$ by hypothesis, and hence $a^{(k / k+1) \circ} \geq f(x)^{(k / k+1) \circ}$ by $\left(b_{2}\right)$. It follows from $\left(b_{1}\right)$ that

$$
a^{(k / k+1) \circ} \circ f\left(x_{k+1}\right)^{(1 / k+1) \circ} \geq f(x)^{(k / k+1) \circ} \circ f\left(x_{k+1}\right)^{(1 / k+1) \circ} .
$$

Therefore we have from Lemma 2 that

$$
\begin{aligned}
\left(f\left(x_{1}\right) \circ \cdots \circ f\left(x_{k+1}\right)\right)^{(1 / k+1) \circ} & =\left(a^{(k) \circ} \circ f\left(x_{k+1}\right)\right)^{(1 / k+1) \circ} \\
& =a^{(k / k+1) \circ \circ f\left(x_{k+1}\right)^{(1 / k+1) \circ}} \\
& \geq f(x)^{(k / k+1) \circ} \circ f\left(x_{k+1}\right)^{(1 / k+1) \circ} \\
& \geq f\left(x^{(k / k+1) *} * x_{k+1}^{(1 / k+1) *}\right) \\
& =f\left(\left(x_{1} * \cdots * x_{k} * x_{k+1}\right)^{(1 / k+1) *}\right) .
\end{aligned}
$$


In other words, the lemma holds for $n=k+1$. Then, by mathematical induction, the lemma holds for all $n \in \mathbf{N}$.

Lemma 4 The inequality

$$
f\left(x_{1}^{\left(p_{1}\right)_{*}} * \cdots * x_{n}^{\left(p_{n}\right) *}\right) \leq f\left(x_{1}\right)^{\left(p_{1}\right)} \circ \cdots \circ f\left(x_{n}\right)^{\left(p_{n}\right) \circ}
$$

holds for all $x_{1}, \ldots, x_{n} \in I, n \in \mathbf{N}$ and $p_{1}, \ldots, p_{n} \in \mathbf{Q}_{+}$with $p_{1}+\cdots+p_{n}=1$.

Proof This result follows directly from Lemma 3.

We are now in a position to prove Theorem 1.

Proof Let $n \in \mathbf{N}, x_{1}, \ldots, x_{n} \in I$ and $t_{1}, \ldots, t_{n} \in \mathbf{R}_{+}$with $t_{1}+\cdots+t_{n}=1$. For each $1 \leq i \leq n$, choose a sequence $\left\{p_{i k}\right\}_{k=1}^{\infty}$ in $\mathbf{Q}_{+}$which converges to $t_{i}$. Put $q_{k}=p_{1 k}+\cdots+p_{n k}$ for each $k \in \mathbf{N}$. Then we have from Lemma 4 that

$$
f\left(x_{1}^{\left(p_{1 k} / q_{k}\right) *} * \cdots * x_{n}^{\left(p_{n k} / q_{k}\right) *}\right) \leq f\left(x_{1}\right)^{\left(p_{1 k} / q_{k}\right)_{\circ}} \circ \cdots \circ f\left(x_{n}\right)^{\left(p_{n k} / q_{k}\right)_{\circ}} .
$$

Hence, after taking the limit with respect to $k$ in (7), we obtain the desired inequality:

$$
f\left(x_{1}^{\left(t_{1}\right) *} * \cdots * x_{n}^{\left(t_{n}\right)_{*}}\right) \leq f\left(x_{1}\right)^{\left(t_{1}\right)_{\circ}} \circ \cdots \circ f\left(x_{n}\right)^{\left(t_{n}\right)_{\circ}} .
$$

Of course, if $f$ is $(*, \circ)$-concave, the above inequality is reversed, as stated in Remark 4 . This completes the proof of Theorem 1.

\section{Applications}

Let $K$ be a topological ordered space with order $\leq$, and let $\circ, *$ and $\diamond$ be three operations in $\mathcal{A}_{+}^{0}(K, \leq)$ which have the following properties:

$$
\begin{aligned}
& (a \circ b) * c=(a * c) \circ(b * c) \quad(\forall a, b, c \in K), \\
& \exists e \in K: e * x=x \quad(\forall x \in K), \\
& a \diamond b=a \circ b \circ(a * b) \quad(\forall a, b \in K) .
\end{aligned}
$$

In this case, it is clear that an element $e$ in (9) is unique.

Lemma 5 The equality $x^{(t)_{\diamond}} \circ e=(x \circ e)^{(t)_{*}}$ holds for each $x \in K$ and $t \in \mathbf{R}_{+}$.

Proof Take $x \in K$ arbitrarily. By (8) and (9), we have $(a \diamond b) \circ e=(a \circ e) *(b \circ e)$ for all $a, b \in K$. By mathematical induction, we have

$$
\left(x_{1} \diamond \cdots \diamond x_{k}\right) \circ e=\left(x_{1} \circ e\right) * \cdots *\left(x_{k} \circ e\right)
$$

for all $k \in \mathbf{N}$ and $x_{1}, \ldots, x_{k} \in K$. In particular, we have

$$
x^{(k)_{\diamond}} \circ e=(x \circ e)^{(k) *}
$$


for all $k \in \mathbf{N}$. Then we have

$$
\begin{aligned}
(x \circ e)^{(n / m)_{*}} & =\left((x \circ e)^{(n)_{*}}\right)^{(1 / m)_{*}} \\
& =\left(x^{(n)_{\diamond}} \circ e\right)^{(1 / m)_{*}} \text { by }(12) \\
& =\left(\left(x^{(n / m)_{\diamond}}\right)^{(m)_{\diamond}} \circ e\right)^{(1 / m)_{*}} \\
& =\left(\left(x^{(n / m)_{\diamond}} \circ e\right)^{(m)_{*}}\right)^{(1 / m)_{*}} \quad \text { by }(12) \\
& =x^{(n / m)_{\diamond}} \circ e
\end{aligned}
$$

for all $n, m \in \mathbf{N}$. Therefore $x^{(p)_{\diamond}} \circ e=(x \circ e)^{(p)_{*}}$ holds for all $p \in \mathbf{Q}_{+}$. Take $t \in \mathbf{R}_{+}$arbitrarily, and choose a sequence $\left\{p_{n}\right\}$ in $\mathbf{Q}_{+}$which converges to $t$. Then

$$
x^{(t)_{\diamond}} \circ e=\lim _{n \rightarrow \infty} x^{\left(p_{n}\right)_{\diamond}} \circ e=\lim _{n \rightarrow \infty}(x \circ e)^{\left(p_{n}\right)_{*}}=(x \circ e)^{(t)_{*}}
$$

holds and so the proof is complete.

Lemma 6 Suppose that $M_{*}(a, b) \leq M_{\circ}(a, b)$ holds for all $a, b \in K$. Then $M_{*}(a, b) \leq$ $M_{\diamond}(a, b) \leq M_{\diamond}(a, b)$ holds for each $a, b \in K$.

Proof Let $a, b \in K$. We first show that $M_{*}(a, b) \leq M_{\diamond}(a, b)$. Since

$$
\left((a * b)^{(1 / 2) *)^{(2) \circ} \leq a \circ b}\right.
$$

it follows from Lemma 5 that

$$
\begin{aligned}
& \left((a * b)^{(1 / 2)_{*}} \circ e\right)^{(2) *}=(a * b) \circ\left((a * b)^{(1 / 2)_{*}}\right)^{(2) \circ} \circ e \\
& \leq(a * b) \circ(a \circ b) \circ e \\
& =(a \diamond b) \circ e \\
& =\left((a \diamond b)^{(1 / 2)_{\diamond}}\right)^{(2)_{\diamond}} \circ e \\
& =\left((a \diamond b)^{(1 / 2)_{\diamond}} \circ e\right)^{(2)_{*}} \text {. }
\end{aligned}
$$

Therefore we obtain from $\left(b_{2}\right)$ for $*$ that

$$
(a * b)^{(1 / 2) *} \circ e \leq(a \diamond b)^{(1 / 2)_{\diamond}} \circ e .
$$

Canceling $e$ in the above inequality, we obtain the desired inequality.

We next show that $M_{\diamond}(a, b) \leq M_{\diamond}(a, b)$. Since

$$
a * b \leq\left((a \circ b)^{(1 / 2) \circ}\right)^{(2)_{*}},
$$

it follows from Lemma 5 that

$$
\begin{aligned}
\left((a \diamond b)^{(1 / 2)_{\diamond}} \circ e\right)^{(2)_{*}} & =\left((a \diamond b)^{(1 / 2)_{\diamond}}\right)^{(2)_{\diamond}} \circ e \\
& =(a \diamond b) \circ e
\end{aligned}
$$




$$
\begin{aligned}
& =(a * b) \circ a \circ b \circ e \\
& \leq\left((a \circ b)^{(1 / 2) \circ}\right)^{(2) *} \circ a \circ b \circ e \\
& =\left((a \circ b)^{(1 / 2) \circ \circ e)^{(2) *} .}\right.
\end{aligned}
$$

Therefore we obtain from $\left(b_{2}\right)$ for $*$ that

$$
(a \diamond b)^{(1 / 2) \diamond} \circ e \leq(a \circ b)^{(1 / 2) \circ} \circ e .
$$

Canceling $e$ in the above inequality, we obtain the desired inequality and so the proof is complete.

The following result is a refinement of the mean inequality.

Theorem 2 Let $K$ be a topological ordered space with order $\leq$ and $*, \circ, \diamond \in \mathcal{A}_{+}^{0}(K, \leq)$ satisfying (8), (9) and (10). If $M_{*}(x, y) \leq M_{\circ}(x, y)$ holds for all $x, y \in K$, then

$$
x_{1}^{\left(t_{1}\right)_{*}} * \cdots * x_{n}^{\left(t_{n}\right)_{*}} \leq x_{1}^{\left(t_{1}\right)_{\diamond}} \diamond \cdots \diamond x_{n}^{\left(t_{n}\right)_{\diamond}} \leq x_{1}^{\left(t_{1}\right)_{\diamond}} \circ \cdots \circ x_{n}^{\left(t_{n}\right) 。}
$$

holds for all $n \in \mathbf{N}, x_{1}, \ldots, x_{n} \in K$ and $t_{1}, \ldots, t_{n} \in \mathbf{R}_{+}$with $t_{1}+\cdots+t_{n}=1$.

Proof This follows immediately from Theorem 1 and Lemma 6.

\section{Examples}

Throughout this section, let $\mathbf{R}_{+}$be an ordinary topological ordered space.

Example 1 Put $x \circ_{t} y=\left(x^{t}+y^{t}\right)^{1 / t}$ for each $x, y \in \mathbf{R}_{+}$and $t \in \mathbf{R} \backslash\{0\}$. Then each $o_{t}$ is a topological abelian semigroup operation on $\mathbf{R}_{+}$such that $x^{(n)_{\circ}}=n^{1 / t} x$ and $x^{(1 / n)_{\circ t}}=(1 / n)^{1 / t} x$ for all $n \in \mathbf{N}$ and $x \in \mathbf{R}_{+}$. Also, since

$$
x^{(n / m)_{\circ} t}=\left(x^{(1 / m)_{\circ} t}\right)^{(n)_{\circ}}=n^{1 / t}(1 / m)^{1 / t} x=(n / m)^{1 / t} x
$$

for each $m, n \in \mathbf{N}, t \in \mathbf{R} \backslash\{0\}$ and $x \in \mathbf{R}_{+}$, it follows that $o_{t} \in \mathcal{A}_{+}^{0}\left(\mathbf{R}_{+}, \leq\right)$for each $t \in \mathbf{R} \backslash\{0\}$. Let $x_{1}, \ldots, x_{n}, \alpha_{1}, \ldots, \alpha_{n} \in \mathbf{R}_{+}$with $\alpha_{1}+\cdots+\alpha_{n}=1$. Since $x^{(\alpha)_{o t}}=\alpha^{1 / t} x$ for each $x, \alpha \in \mathbf{R}_{+}$and $t \in \mathbf{R} \backslash\{0\}$, we have that

$$
x_{1}^{\left(\alpha_{1}\right)_{\circ t}} \circ_{t} \cdots \circ_{t} x_{n}^{\left(\alpha_{n}\right)_{\circ t}}=\left(\alpha_{1} x_{1}^{t}+\cdots+\alpha_{n} x_{n}^{t}\right)^{1 / t}
$$

for all $t \in \mathbf{R} \backslash\{0\}$. Note that

$$
M_{\circ s}(x, y)=\left(\frac{x^{s}+y^{s}}{2}\right)^{1 / s} \leq\left(\frac{x^{t}+y^{t}}{2}\right)^{1 / t}=M_{\circ t}(x, y)
$$

for all $x, y \in \mathbf{R}_{+}$and $s, t \in \mathbf{R} \backslash\{0\}$ with $s \leq t$. Therefore Theorem 1 implies the following well-known inequality:

$$
\left(\alpha_{1} x_{1}^{s}+\cdots+\alpha_{n} x_{n}^{s}\right)^{1 / s} \leq\left(\alpha_{1} x_{1}^{t}+\cdots+\alpha_{n} x_{n}^{t}\right)^{1 / t}
$$

where $s, t \in \mathbf{R} \backslash\{0\}$ with $s \leq t$. 
Example 2 Let $t$ be a positive real number, and let $o_{t}$ be the operation on $\mathbf{R}_{+}$defined in Example 1. Then we have $o_{t} \in \mathcal{A}_{+}^{0}\left(\mathbf{R}_{+}, \leq\right)$. Also, if $x * y=x y\left(x, y \in \mathbf{R}_{+}\right)$, then $* \in \mathcal{A}_{+}^{0}\left(\mathbf{R}_{+}, \leq\right)$ and $x^{(\alpha)_{*}}=x^{\alpha}$ for all $x, \alpha \in \mathbf{R}_{+}$. Hence we have

$$
M_{*}(x, y)=\sqrt{x y} \leq\left(\frac{x^{t}+y^{t}}{2}\right)^{1 / t}=M_{\circ t}(x, y)
$$

for each $x, y \in \mathbf{R}_{+}$.

Let $n \in \mathbf{N}$ and $x_{1}, \ldots, x_{n}, \alpha_{1}, \ldots, \alpha_{n} \in \mathbf{R}_{+}$with $\alpha_{1}+\cdots+\alpha_{n}=1$. Then it is clear that

$$
x_{1}^{\left(\alpha_{1}\right) *} * \cdots * x_{n}^{\left(\alpha_{n}\right) *}=x_{1}^{\alpha_{1}} \cdots x_{n}^{\alpha_{n}}
$$

If $x \diamond y=x \circ_{t} y \circ_{t}(x * y)\left(x, y \in \mathbf{R}_{+}\right)$, then $\diamond \in \mathcal{A}_{+}^{0}\left(\mathbf{R}_{+}, \leq\right)$and three operations $*$, $\circ$ and $\diamond$ on $\mathbf{R}_{+}$satisfy (8), (9) and (10). If $x, \alpha \in \mathbf{R}_{+}$, we have from Lemma 5 that

$$
x^{(\alpha)_{\diamond}} \circ_{t} 1=\left(x \circ_{t} 1\right)^{(\alpha)_{*}}=\left(x \circ_{t} 1\right)^{\alpha}=\left(x^{t}+1\right)^{\alpha / t} .
$$

Then we have from (11) and (15) that

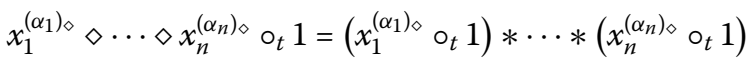

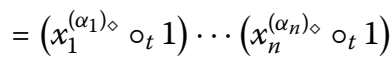

$$
\begin{aligned}
& =\left(x_{1}^{t}+1\right)^{\alpha_{1} / t} \cdots\left(x_{n}^{t}+1\right)^{\alpha_{n} / t} \text {, }
\end{aligned}
$$

and hence

$$
x_{1}^{\left(\alpha_{1}\right)_{\diamond}} \diamond \cdots \diamond x_{n}^{\left(\alpha_{n}\right)_{\diamond}}=\left(\left(x_{1}^{t}+1\right)^{\alpha_{1}} \cdots\left(x_{n}^{t}+1\right)^{\alpha_{n}}-1\right)^{1 / t} .
$$

Then we obtain from (13), (14), (16) and Theorem 2 that

$$
x_{1}^{\alpha_{1}} \cdots x_{n}^{\alpha_{n}} \leq\left(\left(x_{1}^{t}+1\right)^{\alpha_{1}} \cdots\left(x_{n}^{t}+1\right)^{\alpha_{n}}-1\right)^{1 / t} \leq\left(\alpha_{1} x_{1}^{t}+\cdots+\alpha_{n} x_{n}^{t}\right)^{1 / t} .
$$

This is a refinement of the geometric-arithmetic mean inequality.

Competing interests

The authors declare that they have no competing interests.

\section{Authors' contributions}

YN carried out the design of the study and performed the analysis. ST participated in the sequence alignment and drafted the manuscript. All authors read and approved the final manuscript.

\section{Author details}

${ }^{1}$ The Open University of Japan, Chiba, 261-8586, Japan. ${ }^{2}$ Toho University, Funabashi 274-8510, Japan.

\section{Acknowledgements}

The authors are grateful to the referee for careful reading of the paper and for helpful suggestions and comments. The second author is partially supported by Grant-in-Aid for Scientific Research, Japan Society for the Promotion of Science. 


\section{References}

1. Nakasuji, Y, Kumahara, K, Takahasi, S-E: A new interpretation of Jensen's inequality and geometric properties of $\varphi$-means. J. Inequal. Appl. 2011, 48 (2011)

2. Tsurumi, K, Takahasi, S-E, et al.: Fukusokaisekigaku. Shokodo CO., LtD (1988) (Japanese)

3. Craigen, R, Pales, Z: The associativity equation revisited. Aequ. Math. 37, 306-312 (1989)

4. Aczel, J: Sur les opérations définies pour nombres réels. Bull. Soc. Math. Fr. 76, 59-64 (1949)

5. Aczel, J: The state of the second part of Hilbert's fifth problem. Bull. Am. Math. Soc. 20, 153-163 (1989)

doi:10.1186/1029-242X-2013-408

Cite this article as: Nakasuji and Takahasi: A reconsideration of Jensen's inequality and its applications. Journal of Inequalities and Applications 2013 2013:408.

Submit your manuscript to a SpringerOpen ${ }^{\circ}$ journal and benefit from:

- Convenient online submission

- Rigorous peer review

- Immediate publication on acceptance

- Open access: articles freely available online

- High visibility within the field

- Retaining the copyright to your article 